\title{
Obesity and obstructive sleep apnea, bariatric surgery and follow-up post treatment
}

\author{
Won Hee Seo ${ }^{1}$, Christian Guilleminault ${ }^{2^{\star}}$ \\ ${ }^{1}$ Department of Pediatrics, Korea University Ansan Hospital, Korea University College of Medicine, Seoul, Korea \\ ${ }^{2}$ Stanford University Sleep Medicine Division, Redwood City, USA; ${ }^{*}$ Corresponding Author: cguil@stanford.edu
}

Received 18 May 2013; revised 19 June 2013; accepted 15 July 2013

Copyright (C) 2013 Won Hee Seo, Christian Guilleminault. This is an open access article distributed under the Creative Commons Attribution License, which permits unrestricted use, distribution, and reproduction in any medium, provided the original work is properly cited.

\section{ABSTRACT}

Bariatric surgery is a valid treatment alternative for obese patients with obstructive sleep apnea (OSA), but subjects who are overweight or obese represent a poor model to investigate the role of OSA treatment on cardio-vascular variables, calling into question the attribution of cardio-vascular and metabolic problems associated with OSA with excess weight. Seventeen patients with significant obesity who demonstrated OSA with a high apnea-hypopnea index (AHI) were treated with bariatric surgery alone after refusal of nasal continuous positive airway pressure (CPAP) treatment. At approximately 3 years post-surgery, subjects demonstrated a significant drop in blood pressure measured at rest, improvement in sleepiness and fatigue visual analogue scales, but continued to complain of daytime sleepiness. Polysomnography in these subjects demonstrated relative improvement in $\mathrm{AHI}$, but patients continued to have a significant number of respiratory events and episodes of flow limitation. Subsequent treatment with a dental device led to further improvement in nocturnal respiration, but did not reduce $A H I$ to a normal range on repeat polysomnography. In this study we demonstrate that bariatric surgery alone and in conjunction with a dental device may significantly reduce $\mathrm{AHI}$ but does not eliminate daytime fatigue and sleepiness. Systematic long term follow-up examining the persistence of sleep related abnormalities should be performed particularly if daytime fatigue or sleepiness is still present in patients with OSA after treatment with bariatric surgery alone.

Keywords: Obstructive Sleep Apnea; Obesity;
Bariatric Surgery; Blood Pressure; Flow Limitation

\section{INTRODUCTION}

Obesity is associated with obstructive sleep apnea. The pattern of obstructive sleep apnea (OSA) was first described in a Pickwickian patient in 1960 by Gerardy, et al. [1]. In 1972, Guilleminault, et al. emphasized that normal weight subjects presented with the same constellation of clinical symptoms and polysomnographic pattern and coined the term obstructive sleep apnea syndrome (OSAS), and then terms subjects with OSA [2,3]. With the occurrence of the obesity epidemic, a large number of subjects have been seen again with both obesity and OSA. Obesity leads to similar cardio-vascular and inflammatory changes as OSA does in non-obese subjects, and fMRI studies have shown that pre-frontal cognitive functions can be affected by both conditions. There have been controversies concerning the respective role of obesity and OSA on many aspects of health. For example, insulin resistance has been found to be unrelated to OSA in normal weight subjects while there has been an association found between insulin resistance and obese patients with OSA. In modern times, very obese patients are submitted to bariatric surgery. Usually surgeons examine patients to evaluate risk of OSA before performing the surgery and may refer them for nasal continuous positive airway pressure (CPAP) treatment. However many patients are non-compliant with nasal CPAP and some patients refuse treatment other than abdominal surgery.

This retrospective analysis reports on 17 patients with obesity-OSA who were diagnosed at the time of bariatric surgery, and subsequently followed for 3 years postsurgery. Despite weight loss, these patients continued to endorse some degree of sleepiness and fatigue, and were referred back to the sleep clinic where a new PSG was performed. If patients continued to refuse use of positive 
airway pressure treatment a dental device was prescribed and a 3rd evaluation was performed approximately 2 months after the initiation of treatment.

\section{MATERIALS AND METHODS}

Patients were seen initially by bariatric surgery services for evaluation and decision on whether they represented good surgical candidates. All patients accepted for surgery after clinical evaluation, were referred to the sleep laboratory preoperatively for determination of the presence of associated OSA and its severity. Patients were provided with results of the sleep test and nasal CPAP was recommended. Patients had either a trial of nasal CPAP and refused to pursue treatment or refused to use nasal CPAP from the start. Patients then underwent bariatric treatment.

\subsection{Baseline Investigation}

Patients presented at the sleep laboratory at 1800 hours. Demographics including weight and height, bodymass-index (BMI) and neck circumference were obtained. At that time subjects filled out questionnaires regarding their daytime activities and sleep disorders [4]. They also completed two visual analogue scales (VAS), in which they traced a vertical line on a $100 \mathrm{~mm}$ horizontal line to mark their subjective rating of "sleepiness" and "fatigue". Subjects were asked to consider their degree of sleepiness and fatigue during the previous day and then traced a line between "wide awake all day long” (0) and "extremely sleepy, having to continuously fight sleep all day long” (100), for "sleepiness" and from "no fatigue all day long" (0), to "extreme fatigue during the entire day" (100) for "fatigue".

While in the sleep laboratory between 1800 and 1900 hours, subjects were kept seated at rest for 30 minutes in a quiet environment and then blood pressure was measured twice 5 minutes apart, and the mean of the 2 systolic and diastolic measurements was collected in the subject information chart.

The initial determination of the presence or absence of OSA was performed as a home study using a 4 channel recorder. The following variables were monitored: nasal cannula pressure transducer, pulse oximetry, one lead electrocardiography (ECG) and neck microphone. Patients were given a sleep log where they indicated bed time, any bathroom awakening or other long awakening during the night, and awakening time. A minimum of 5 hours of appropriate nocturnal recording was requested or the test was repeated. Subjects also pushed a button that marked their bed-time and final awakening on the recording. The equipment was placed on subjects in the sleep laboratory in the evening.

Each recording was manually scored for "apneas and hypopneas" following the recommendations of the American Academy of Sleep Medicine, and lowest oxygen saturation was noted after elimination of artifactual segments [5].

Patients were provided with results of the sleep test and initiation of treatment with nasal CPAP was recommended. Patients either attempted CPAP use and later discontinued, or refused to use nasal CPAP from the start. Patients then underwent bariatric treatment involving a Roux en Y procedure.

\subsection{Follow-up Evaluation}

Patients were regularly followed-up by the surgery clinic, and demonstrated positive surgical results with improvement of BMI and weight. However, some patients still complained of "daytime fatigue" or" sleepiness" and these subjects were re-evaluated in sleep clinic.

These select patients underwent a full nocturnal PSG between 31 and 37 months after surgery. Patients came to the sleep laboratory again between 1800 and 1900 hours and demographic information was again obtained. The subjects filled out the Sleep Disorders Questionnaire, the sleepiness and fatigue visual analogue scales, and their blood pressure was taken the same way as described during the initial visit.

During this PSG the following variables were monitored: 4 EEG leads, two electrooculoragraphy (EOG) leads, one chin and two leg electromyography (EMG) leads, one electrocardiography (ECG) lead. Respirations were monitored with a nasal cannula pressure transducer, an oral thermistor, thoracic and abdominal inductive plethysmography bands, pulse-oximetry, a neck microphone and a body position sensor. Video-monitoring was performed during the recording.

Results were again provided to patients, again some of whom refused nasal CPAP treatment.

\subsection{Dental Device Treatment}

Patients were referred to an orthodontist specialized in fitting dental devices of their choice for the treatment of sleep-disordered breathing (SDB). The device was fitted by orthodontist and all patients were fitted with a 'bi-block" dental device that may have needed adjustment and titration during the first weeks. Patients were sent back to the sleep laboratory for a repeat PSG after two months of dental device usage. During the third examination, the same procedures as those used during the two first visits were performed. Patients presented between 1800 and 1900 hours, had repeat demographic measurements and filled out the same questionnaires and visual analogue scales. They underwent a nocturnal PSG in which the same variables as mentioned above were monitored. 


\subsection{Data Analysis}

The retrospective analysis of collected clinical data rendered anonymous was approved by the local IRB: The previously collected data were rendered anonymous before analyses, and were re-tabulated. The data from each subject were collected by an individual unaware of the status of the patient at the time of the data collection.

\subsection{Statistics}

Comparison between variables (AHI, lowest $\mathrm{SpO}_{2}, \mathrm{BP}$ and VAS scores) was performed by one-way repeated analysis of variance (ANOVA). For post hoc analysis, a Student Newman-Keuls Test was used. For BMI and neck circumference, measurements from pre-to post-bariatric surgery were analyzed using paired t test with Wilcoxon Signed-Rank Test. All analyses were conducted using SAS software version 9.2 (SAS Institute Inc, Cary, NC, USA), and a two-sided P-value $<0.05$ was considered to be statistically significant.

\section{RESULTS}

\subsection{Baseline}

All patients were male with a mean age $34.6 \pm 4.59$ (mean \pm SD, range 27-43). All subjects were obese. Pa- tient VAS results showed presence of sleepiness and fatigue. None of the patients carried the diagnosis of hypertension, but blood pressures at rest seated showed abnormal systolic and diastolic values in all cases (Table 1).

Ambulatory monitoring howed the presence of abnormal breathing during sleep with a clearly abnormal apnea-hypopnea index (AHI) and with minimum oxygen $\left(\mathrm{O}_{2}\right)$ saturations of $81 \%$ or less. The demographic results are presented in Table 1.

\subsection{Follow-up Evaluation}

As mentioned, despite the review of sleep data and the recommendation of nasal CPAP, these select patients refused treatment, preferring instead to be treated for their obesity with surgery. Patients were followed by the surgery clinic for 3 years and all had significant weight loss (Table 2), but also still had complained of daytime fatigue and/or sleepiness. The interviews, questionnaires and VAS indicated presence of some degree of fatigue and sleepiness, despite clear improvements compared to initial visit.

All patients completed another nocturnal recording in the form of an in-laboratory PSG. As shown in Table 3, there was persistence of OSA with an elevated AHI

Table 1. Demographic, respiratory, cardiologic data during sleep in 17 subjects.

\begin{tabular}{|c|c|c|c|c|c|c|c|c|c|c|}
\hline Subjects & $\begin{array}{c}\text { age } \\
\text { (year) }\end{array}$ & sex & $\begin{array}{c}\mathrm{BMI}^{\mathrm{a}} \\
\left(\mathrm{kg} / \mathrm{m}^{2}\right)\end{array}$ & $\begin{array}{c}\text { Neck } \\
\text { circumference }(\mathrm{cm})\end{array}$ & $\begin{array}{c}\mathrm{AHI}^{\mathrm{b}} \\
\text { (events/h) }\end{array}$ & $\begin{array}{c}\text { Lowest } \\
\mathrm{SpO}_{2}(\%)\end{array}$ & $\begin{array}{l}\text { Systolic BP } \\
\text { (mmHg) }\end{array}$ & $\begin{array}{l}\text { Diastolic BP } \\
\text { (mmHg) }\end{array}$ & $\begin{array}{l}\text { VAS }^{\mathrm{d}} \text { for Sleepiness } \\
(0-100)\end{array}$ & $\begin{array}{c}\text { VAS for Fatigue } \\
(0-100)\end{array}$ \\
\hline 1 & 37 & $\mathrm{~m}$ & 43 & 51 & 31 & 79 & 180 & 100 & 57 & 74 \\
\hline 2 & 29 & $\mathrm{~m}$ & 45 & 53 & 27 & 81 & 150 & 79 & 59 & 61 \\
\hline 3 & 33 & $\mathrm{~m}$ & 39 & 46 & 35 & 78 & 160 & 86 & 56 & 51 \\
\hline 4 & 42 & $\mathrm{~m}$ & 41.7 & 48 & 43 & 77 & 142 & 89 & 59 & 64 \\
\hline 5 & 27 & $\mathrm{~m}$ & 46 & 49 & 44 & 74 & 138 & 84 & 69 & 78 \\
\hline 6 & 35 & $\mathrm{~m}$ & 38.7 & 47 & 26 & 80 & 155 & 90 & 73 & 81 \\
\hline 7 & 43 & $\mathrm{~m}$ & 43.4 & 44 & 45 & 72 & 163 & 92 & 71 & 80 \\
\hline 8 & 34 & $\mathrm{~m}$ & 40.6 & 46.3 & 43 & 71 & 154 & 91 & 67 & 65 \\
\hline 9 & 32 & $\mathrm{~m}$ & 43 & 44.6 & 47 & 70 & 141 & 83 & 78 & 80 \\
\hline 11 & 30 & $\mathrm{~m}$ & 43.3 & 48.2 & 48 & 73 & 153 & 90 & 57 & 70 \\
\hline 12 & 37 & $\mathrm{~m}$ & 43.8 & 50.2 & 39 & 80 & 164 & 91 & 45 & 64 \\
\hline 13 & 28 & $\mathrm{~m}$ & 45 & 52.3 & 47 & 69 & 148 & 83 & 75 & 80 \\
\hline 14 & 37 & $\mathrm{~m}$ & 40.4 & 45.7 & 39 & 75 & 158 & 92 & 70 & 70 \\
\hline 15 & 34 & $\mathrm{~m}$ & 39 & 46.8 & 46 & 70 & 163 & 89 & 62 & 75 \\
\hline 16 & 35 & $\mathrm{~m}$ & 42.7 & 49.4 & 35 & 76 & 152 & 88 & 55 & 70 \\
\hline 17 & 40 & $\mathrm{~m}$ & 39.7 & 50.4 & 38 & 79 & 169 & 92 & 50 & 72 \\
\hline mean & 34.6 & & 42.3 & 48.2 & 39.5 & 75.3 & 156.3 & 88.9 & 62.8 & 71.2 \\
\hline range & $29-43$ & & $38.7-46$ & $44-53$ & $26-48$ & $69-81$ & $138-180$ & $79-100$ & $45-78$ & $51-81$ \\
\hline
\end{tabular}

${ }^{\mathrm{a}} \mathrm{BMI}$ : body mass index; ${ }^{\mathrm{b}} \mathrm{AHI}$ : Apnea-hypopnea index; ${ }^{\mathrm{c}} \mathrm{BP}$ : blood pressure ; ${ }^{\mathrm{d}} \mathrm{VAS}$ : visual analogues scales. 
Table 2. Baseline demographics, anthropometric characteristics in 17 subjects at baseline and, 3-year follow-up after bariatric surgery.

\begin{tabular}{cccc}
\hline & Baseline & After bariatric surgery & Between differences $(p$-value $)$ \\
\hline Number of subjects & 17 & 17 & $<0.0001$ \\
BMI $\left(\mathrm{kg} / \mathrm{m}^{2}\right)$ & $42.25 \pm 2.31$ & $28.27 \pm 1.19$ & $<0.0001$ \\
Neck circumference $(\mathrm{cm})$ & $48.21 \pm 2.59$ & $40.31 \pm 1.13$ & \\
\hline
\end{tabular}

(11.65 \pm 2.40 event/hour), and “flow limitation” which was noted during a mean $72.53 \%$ of total sleep time. Compared to baseline, however, significant improvements in weight, BMI, neck circumference as well as significantly improved AHI and lowest oxygen saturation were found. In addition, systolic blood pressure was significantly improved with weight loss despite persistence of OSA compared to baseline (Figure 1).

\subsection{Dental Device Treatment}

All patients were fitted with a dental device classified in the general group of "bi-block". Some of the devices needed adjustment during first few weeks. Following completion of adjustment all subjects used the device for 2 months and were then restudied to assess effectiveness.

As seen in Table 3 there was a further decrease in the AHI, however an AHI of less than 5 events per hour of sleep (normal) was not obtained. During the recording patients also demonstrated decreased "flow limitation", as defined by Hosselet, et al. [6] (Figure 2). The percentage of time spent in "flow limitation" decreased from a mean of $72.54 \%$ of sleep time before dental device application to a mean of 34.9\% (Figure 3), indicating better breathing during sleep despite absence of significant BMI change during the short survey period.

\section{DISCUSSION}

This report outlines 17 subjects with obesity and the presence of OSA upon initial monitoring who underwent successful bariatric surgery but continued to endorse suf-

Table 3. Sleep polysomnographic data and VAS score in 17 subjects at baseline, 3-year follow-up after bariatric surgery and then after dental device.

\begin{tabular}{ccccc}
\hline & Baseline & $\begin{array}{c}\text { After } \\
\text { bariatric } \\
\text { surgery }\end{array}$ & $\begin{array}{c}\text { After } \\
\text { Dental } \\
\text { device }\end{array}$ & $\begin{array}{c}\text { Between } \\
\text { differences } \\
(p \text {-value })\end{array}$ \\
\hline \multicolumn{4}{c}{ Polysomnograpy } \\
AHI (events/h) $39.52 \pm 6.87$ & $11.65 \pm 2.40$ & $7.18 \pm 1.67$ & $<0.0001$ \\
Lowest $\mathrm{O}_{2}(\%)$ & $75.29 \pm 3.93$ & $89.47 \pm 1.01$ & $91.47 \pm 2.00$ & $<0.0001$ \\
& & VAS & score $(0-100)$ & \\
Sleepiness & $62.82 \pm 9.18$ & $41.24 \pm 5.13$ & $25.53 \pm 4.80$ & $<0.0001$ \\
Fatigue & $71.18 \pm 8.18$ & $46.00 \pm 7.16$ & $30.24 \pm 3.56$ & $<0.0001$ \\
\hline
\end{tabular}

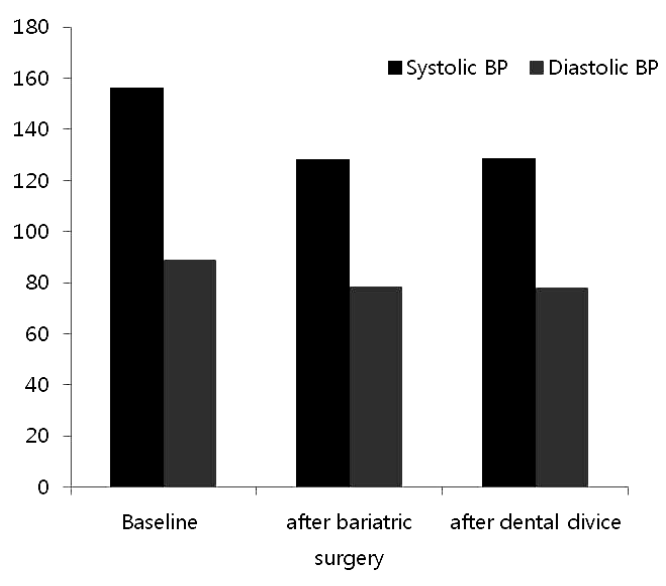

Figure 1. Changes in blood pressure. There are significant improvements after bariatric surgery in systolic blood pressure $(p<0.0001)$.

ficient sleep related complaints to be sent back to sleep clinic for further evaluation.

Our study has several limitations. It is a retrospective study, and carries with it all the problems associated with such an analysis. The subjects were also self-selected and were sent back to the sleep laboratory due to their persistent complaints of daytime fatigue and/or sleepiness. Our investigation is thus not a systematic longitudinal study of all patients undergoing bariatric surgery during a specific time frame. Finally patients were followed by bariatric surgeons and the data presented here was obtained in the sleep laboratory only.

In addition, ambulatory monitoring was employed upon entry into the study, while full PSGs were performed only for second and third recordings. Despite these limitations, our retrospective investigation includes important findings that further our understanding of OSA in overweight and obese patients

There have been controversies regarding obese patients who are found to also have OSA. Patients with obesity will have enlarged tongues due to fat deposits [7], and obstructive sleep apnea syndrome (OSAS) noted in these subjects can often be related to a secondary narrowing of the upper airway due to the fatty infiltration of the different soft tissue structures within the upper airway. As early as 1986, Jamieson, et al. had shown that overweight and obese patients had, in general, a wider oral-facial anatomy when compared to non-overweight OSA subjects [8]. This study suggested that obesity may 


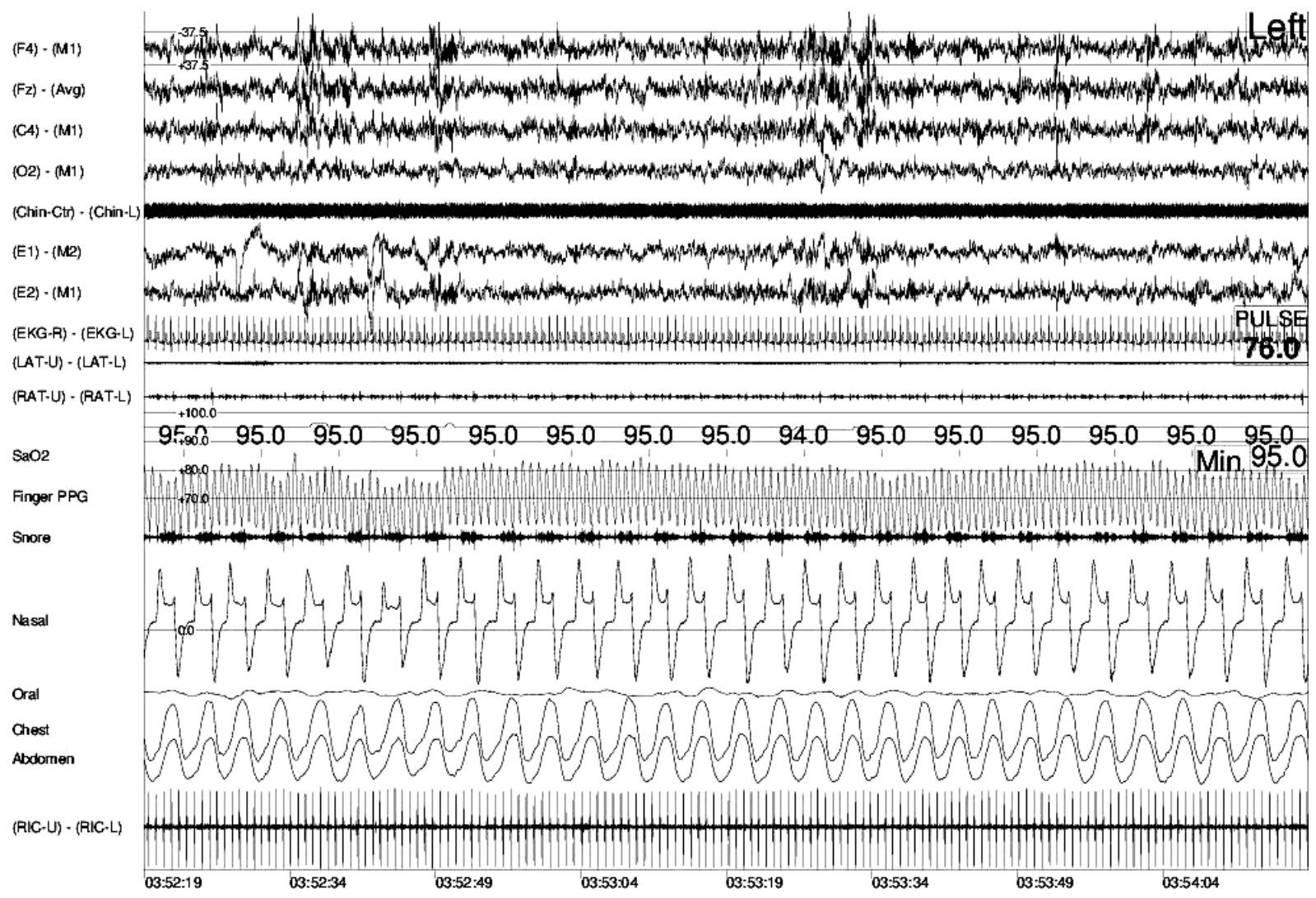

Figure 2. Polysomographic recording of flow limitation during NREM sleep in a patient with dental device. Channels from top: 4 electroencephalography (EEG) leads, 1 chin electromyography (EMG) leads, 2 electro-oculragraphy (EOG) leads, one electrocardiography (ECG), 2 leg EMG pulseoximetry, figerplethysmography, neck microphone, nasal cannula pressure transducer, oral thermistor, chest and abdominal plethysmography signals, diaphragmatic/intercostal EMG. As shown on lead 14 from top, the nasal cannula channel shows "flow limitation" with an abnormal curve of the flow: instead of having a clear sinusoidal curve the flow during the inspiratory phase is truncated (Black arrow).

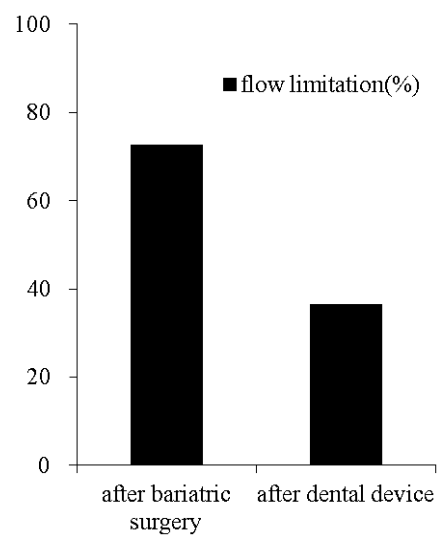

Figure 3. Comparison between after bariatric surgery and dental device for percentage of flow limitation. Despite of significant decreasing percentage of flow limitation, there is still persistent flow limitation $(p<0.0001)$.

occur before development of any upper airway sleep disordered breathing in some patients.
Many of the complications and co-morbidities associated with OSAS are also linked to obesity such as the association with insulin resistance [9] or the reported cognitive dysfunction with impairments of memory and attention [10-12].

Controversy remains in linking certain cardio-vascular co-morbidities, particularly hypertension, to OSA independent of obesity [13]. Our study demonstrates that the sole treatment of obesity leads to both a significant decrease in OSA and hypertension in many subjects (Figure 1). These results were obtained without nasal CPAP intervention.

Many of the studies emphasizing that cardio-vascular and metabolic abnormalities are related to OSA have been performed on subjects with a BMI of at least 28 $\mathrm{kg} / \mathrm{m}^{2}$, which is the inferior limit of obesity as defined by the World Health Organization [14,15], Before attributeing specific co-morbidities to OSAS, more important attention must be paid to the isolated role of abnormal weight and the specific role of the adipocyte proliferation that is always associated with obesity. The peptides controlled by the hyperactive adipocytes in an overweight/ 
obese subject may explain, many of the co-morbidities attributed to OSA in overweight individuals.

Our retrospective analysis shows that bariatric surgery is an approach that is successful in reducing OSA as demonstrated by PSG. It also shows that bariatric surgery significantly lowers blood pressure without nasal CPAP. The treatment of obesity alone has a significant impact on blood pressure, which is something well-known but not emphasized enough.

When high BMI or obesity are present, positive changes such as decrease in blood pressure, obtained after usage of nasal CPAP or bi-level often are inappropriately considered as secondary evidence for the role of OSA in the onset of the initially recorded abnormal blood pressure: But obesity per se induces many cardio-vascular changes, including induction of high blood pressure (HBP), without having to evoke presence of OSA, furthermore PAP equipment deals not only with the upper airway but also with the chest-bellows problem related to overweight/obesity status, further limiting attribution of initially observed HBP to OSA.

Greenberg, et al. reported a meta-analysis of the response to bariatric surgery and showed that that such treatment does not always completely relieve symptoms associated with abnormal breathing during sleep [16]. In the present study, there was a significant improvement compared to baseline in the VAS scores, but subjectively patients complained enough for their surgeons to request additional sleep evaluation. Several factors may be causing these residual sleep complaints. Subjects may still have consequences of their previous overweight status, since none of our subjects had a BMI below $25 \mathrm{~kg} / \mathrm{m}^{2}$. Also, the effect known as "blunting of the arousal response" occurs with sleep fragmentation, and this blunting will lessen with a decrease in the frequency of sleep fragmentation [17]. As a result, patients with an improved BMI after surgery may paradoxically perceive more subjective sleep disruption as blunting of arousal response may not be as important as at baseline due to better sleep with simultaneous decrease in sleep fragmentation.

Further success was noted with use of a dental device, but again only partial improvements were seen as there was persistent "flow limitation" even after the use of a dental device (Figure 3).

Flow limitation pattern is associated with a clear increase in the frequency of phase A2 and A3 of the cyclic-alternating-pattern (CAP) as can be seen in the EEG leads in Figure 2 [18]. This increase in frequency of phases A2 and A3 of CAP during total NREM sleep time is indicative of sleep disruption associated with complaints of daytime tiredness, fatigue and sleepiness [14]. In OSA patients, despite initially reported improvement of sleepiness and fatigue with a dental device, OSA pa- tients may complain again in the future of "worsening" of their daytime status. "Flow limitation" is not recognized in many sleep-scoring manual despite its establishment as a pathological event since 1999 [6]. Several investigations have shown that "flow-limitation may be as detrimental to daytime well-being as presence of apneas and hypopneas when comparing complaints of fatigue, tiredness and sleepiness [19, 20].

Recently, Palombini, et al. using the results of the Sao Paulo epidemiological studies on sleep disorders, gathered a representative sample of the general population and, with collection of 1042 nocturnal PSGs, defined the percentage of flow limitation that should be considered as "abnormal" and placed this percentage at $>30 \%$ of total sleep time [21]. Scoring of flow limitation involve systematic usage of a nasal cannula pressure transducer, the signal from which recorded with a DC amplifier using a specific calibration. These data will help to better defined abnormal SDB and to determine better control of abnormal breathing during sleep.

\section{CONCLUSION}

Bariatric surgery is a valid alternative treatment for patients with obesity and OSA. Here, weight loss was associated with significant blood pressure improvement despite the presence of residual OSA at long term follow-up. However, patients treated with bariatric surgery need regular follow-up from sleep clinics for potential development of apparently unexplained daytime fatigue or sleepiness. Despite significant weight loss, these patients may have oral-facial anatomic features responsible for residual SDB. Finally, overweight/ obese patients and OSA have a complex health problem, and investigation of these patients should not be used to define co-morbidity associated with OSA.

\section{REFERENCES}

[1] Gerardy, W., Herberg, D. and Kuhn, H. (1960) Vergleichende untersuchungen der lungenfunktion und des elektroen-cephalogramms bie zwei patienten mit pickwickiansyndrom. Zeitschrift Fur Klinische Psychologie Psychia- trie Und Psychotherapie, 156, 362-380.

[2] Guilleminault, C., Eldridge, F. and Dement, W. (1972) Insomnia, narcolepsy, and sleep apneas. Bulletin de physio-pathologie respiratoire, $\mathbf{8}, 1127-1138$.

[3] Guilleminault, C., Tilkian, A. and Dement, W.C. (1976) The sleep apnea syndromes. Annual Review of Medicine, 27, 465-484. doi:10.1146/annurev.me.27.020176.002341

[4] Douglass, A.B., Bornstein, R.F., Nino-Murcia, G. and Keenan, S. (1994) The sleep disorders questionnaire: I. Creation and multivariate structure of SDQ. Sleep: Journal of Sleep Research \& Sleep Medicine, 17, 160-167.

[5] Iber, C., Ancoli-Israel, S., Chesson, A. and Quan, S.F. 
(2007) The AASM manual for the scoring of sleep and associated events: Rules, terminology and technical specifications. American Academy of Sleep Medicine, Westchester.

[6] Hosselet, J.J., Norman, R.G., Ayappa, I. and Rapoport, D. (1998) Detection of flow limitation with a nasal cannula/pressure transducer system. American Journal of Respiratory and Critical care Medicine, 157, 1461-1467. doi:10.1164/ajrccm.157.5.9708008

[7] Schwab, R.J., Pasirstein, M., Pierson, R., Mackley, A., Hachadoorian, R., Arens, R., Maislin, G. and Pack, A.I. (2003) Identification of upper airway anatomic risk factors for obstructive sleep apnea with volumetric magnetic resonance imaging. American Journal of Respiratory and Critical Care Medicine, 168, 522-530. doi:10.1164/rccm.200208-8660C

[8] Jamieson, A., Guilleminault, C., Partinen, M. and QueraSalva, M.A. (1986) Obstructive sleep apneic patients have craniomandibular abnormalities. Sleep, 9, 469-477.

[9] Stoohs, R.A., Facchini, F. and Guilleminault, C. (1996) Insulin resistance and sleep-disordered breathing in healthy humans. American Journal of Respiratory and Critical Care Medicine, 154, 170-174. doi:10.1164/ajrccm.154.1.8680675

[10] Wolf, P.A., Beiser, A., Elias, M.F., Au, R., Vasan, R.S. and Seshadri, S. (2007) Relation of obesity to cognitive function: importance of central obesity and synergistic influence of concomitant hypertension. The Framingham Heart Study. Current Alzheimer Research, 4, 111-116. doi:10.2174/156720507780362263

[11] Gazdzinski, S., Millin, R., Kaiser, L.G., Durazzo, T.C., Mueller, S.G., Weiner, M.W. and Meyerhoff, D.J. (2010) $\mathrm{BMI}$ and neuronal integrity in healthy, cognitively normal elderly: A proton magnetic resonance spectroscopy study. Obesity, 18, 743-748. doi:10.1038/oby.2009.325

[12] Cournot, M., Marquie, J., Ansiau, D., Martinaud, C., Fonds, H., Ferrieres, J. and Ruidavets, J.B. (2006) Relation between body mass index and cognitive function in healthy middle-aged men and women. Neurology, 67, 1208-1214. doi:10.1212/01.wnl.0000238082.13860.50
[13] Guilleminault, C. and Stoohs, R. (1995) Arousal, increased respiratory efforts, blood pressure and obstructive sleep apnoea. Journal of Sleep Research, 4, 117-124. doi:10.1111/j.1365-2869.1995.tb00200.x

[14] Tantrakul, V., Park C.S. and Guilleminault, C. (2012) Sleep-disordered breathing in premenopausal women: Differences between younger (less than 30years old) and older women. Sleep Medicine, 13, 656-662. doi:10.1016/j.sleep.2012.02.008

[15] Tantrakul, V. and Guilleminault, C. (2009) Chronic sleep complaints in premenopausal women and their association with sleep-disordered breathing. Lung, 187, 82-92. doi:10.1007/s00408-009-9137-7

[16] Greenberg, I., Perna, F., Kaplan, M. and Sullivan, M.A. (2005) Behavioral and psychological factors in the assessment and treatment of obesity surgery patients. Obesity Research, 13, 244-249. doi:10.1038/oby.2005.33

[17] Guilleminault, C. and Rosekind, M. (1981) The arousal threshold: sleep deprivation, sleep fragmentation, and obstructive sleep apnea syndrome. Bulletin Européen de Physiopathologie Respiratoire, 17, 341-349.

[18] Terzano, M.G., Parrino, L., Sherieri, A., Chervin, R., Chokroverty, S., Guilleminault, C., Hirshkowitz, M., Mahowald, M., Moldofsky, H. and Rosa, A. (2001) Atlas, rules, and recording techniques for the scoring of cyclic alternating pattern (CAP) in human sleep. Sleep medicine, 2, 537-553. doi:10.1016/S1389-9457(01)00149-6

[19] Guilleminault, C., Poyares, D., Rosa, Ad., Kirisoglu, C., Almeida, T.L. and Maria C. (2006) Chronic fatigue, unrefreshing sleep and nocturnal polysomnography. Sleep Medicine, 7, 513-520. doi:10.1016/j.sleep.2006.03.016

[20] Aittokallio T., Saaresranta T., Polo-Kantola P., Nevalainen, O. and Polo, O. (2001) Analysis of inspiratory flow shapes in patients with partial upper-airway obstruction during sleep. Chest, 119, 37-44. doi:10.1378/chest.119.1.37

[21] Palombini, L., Tufik, S., Rapoport, D., Ayappa, I., Guilleminault, C., De Godoy, L., Casto, L. and Bittencourt, L. (2013) Inspiratory flow limitation in a normal population of adults in Sao Paolo, Brazil. Proceeding of Sleep. 